\title{
Metodologia para avaliação dos fatores condicionantes da velocidade em regime livre em ambiente periurbano
}

\author{
António Lobo ${ }^{1}$, Fernanda Sousa ${ }^{2}$, Carlos Rodrigues ${ }^{3}$ e António Couto ${ }^{4}$
}

\begin{abstract}
Resumo: Neste artigo, apresenta-se uma metodologia para a avaliação macroscópica da influência das características geométricas e ambientais e da sinalização de velocidade limite legal na velocidade em regime livre praticada pelos condutores em zonas periurbanas ou de transição. Um condutor circula em regime livre quando a sua escolha de velocidade não é condicionada pela presença do veículo precedente. Assim, a velocidade adotada reflete a sua resposta face às características da estrada e do ambiente envolvente. A metodologia consiste na utilização de métodos estatísticos de análise de dados multivariados, nomeadamente de classificação e de análise em componentes principais, para a definição de variáveis macroscópicas caracterizadoras das zonas periurbanas, sobre as quais é depois efetuada uma análise de regressão que permite a avaliação dos impactos das diferentes variáveis na velocidade em regime livre. A metodologia proposta é aplicada a um conjunto de 27 locais pertencentes a estradas de duas vias situadas em Portugal, onde foram observadas as velocidades praticadas, a existência de sinalização de limite legal de velocidade e diversas variáveis microscópicas caracterizadoras da geometria e do ambiente.
\end{abstract}

Palavras-chave: velocidade em regime livre, ambiente periurbano, análise de dados multivariados.

\begin{abstract}
This paper presents a new methodology for the evaluation of the effects of posted speed limit, geometric and environmental characteristics on free-flow speed in transition zones, through a macroscopic analysis. A vehicle travels under free-flow conditions if its chosen speed is not affected by the presence of the vehicle ahead. In this sense, the free-flow speed reflects the driver's response to road geometric and environmental characteristics. In the first stage of the proposed methodology, new macroscopic variables reflecting the characteristics of transition zones are created through the use of multivariate data analysis techniques, namely analysis of clusters and principal component analysis. In the second stage, a regression analysis is performed in order to compare the effects of different variables on the adopted free-flow speed. This methodology is then tested for a set of 27 sites located in Portuguese two-lane highways, where drivers' speed, the existence of posted speed limit and some geometric and environmental microscopic characteristics were collected.
\end{abstract}

Keywords: free-flow speed, transition zones, multivariate data analysis.

\section{INTRODUÇÃo}

As zonas periurbanas, também designadas por zonas de transição, caracterizam-se por marcarem a passagem entre ambientes rurais e ambientes urbanos, apresentando características intermédias em relação a estes. De facto, uma zona periurbana é geralmente caracterizada pela existência de índices de construção marginal superiores ao ambiente rural - onde a existência de edifícios marginais à estrada é pontual/isolada - sem que, contudo, apresente uma frente urbana consolidada. As necessidades de acessibilidade aumentam com a densidade de construção, pelo que não é de estranhar que as zonas periurbanas apresentem características intermédias para os indicadores que preconizam a acessibilidade de veículos e peões, tais como a densidade de intersecções, passagens pedonais ${ }^{1}$ e passeios. A título de curiosidade, refira-se que uma observação qualitativa sobre a continuidade de passeio pode fornecer uma forte indicação sobre o tipo

\footnotetext{
${ }^{1}$ António Lobo, Centro de Investigação do Território, Transportes e Ambiente. Faculdade de Engenharia da Universidade do Porto, Porto, Portugal. (email: lobo@fe.up.pt).

${ }^{2}$ Fernanda Sousa, Centro de Investigação do Território, Transportes e Ambiente. Faculdade de Engenharia da Universidade do Porto, Porto, Portugal. (email: fcsousa@fe.up.pt).

${ }^{3}$ Carlos Rodrigues, Centro de Investigação do Território, Transportes e Ambiente. Faculdade de Engenharia da Universidade do Porto, Porto, Portugal. (e-mail: cmr@fe.up.pt).

${ }^{4}$ António Couto, Centro de Investigação do Território, Transportes e Ambiente. Faculdade de Engenharia da Universidade do Porto, Porto, Portugal. (email: fcouto@fe.up.pt)

Manuscrito recebido em 17/2/2012 e aprovado para publicação em 10/5/2012. Este artigo é parte de TRANSPORTES v. 20, n. 1, 2012. ISSN: 2237-1346 (online).
}

de ambiente em causa: em zona rural, o passeio é praticamente inexistente; em zona periurbana o passeio é descontínuo e acompanha frequentemente a existência de edifícios (situação muito comum em Portugal); em zona urbana, o passeio é geralmente contínuo.

Situando-se em posição intermédia entre o ambiente rural (limite legal de velocidade de $90 \mathrm{~km} / \mathrm{h}$ ) e o ambiente urbano (limite legal de velocidade de $50 \mathrm{~km} / \mathrm{h}$ ), o ambiente periurbano constitui uma zona de redução de velocidade na aproximação às localidades. No entanto, o Código da Estrada português não estabelece um limite legal intermédio e específico para este tipo de ambiente, que nem sempre consegue transmitir ao condutor a necessidade de redução de velocidade devido à aproximação de uma zona urbana (Schmidt e Tiffin, 1969; Denton, 1976; Matthews, 1978). As zonas de transição são particularmente sensíveis em termos de sinistralidade, registando taxas de ocorrência de acidentes com vítimas bastante superiores às observadas em ambiente rural (Tziotsis, 1992). A publicação produzida pela NCHRP (2011) contempla uma ampla revisão do estado da arte no que concerne ao estudo da velocidade e à implementação de medidas de acalmia de tráfego nas zonas de transição.

Em Portugal, as entidades gestoras da rede viária (governo central e autarquias) têm prestado uma crescente atenção à problemática das velocidades praticadas em ambiente periurbano, através da aplicação de um grande número de medidas corretivas do traçado e de medidas de acalmia de tráfego, tais como: (i) alterações ao traçado em planta (e. g. construção de rotundas ${ }^{2}$ ); (ii) alterações ao traçado em per-

${ }^{1}$ travessias de pedestres (terminologia brasileira). 
fil longitudinal (e. g. implantação de $\operatorname{lombas}^{3}$ de abrandamento); (iii) alterações ao traçado em perfil transversal (e. g. construção de passeios ou separadores centrais); (iv) implementação de sinalização diversa (e. g. sinais de limite de velocidade, semáforos ou bandas cromáticas); (v) melhoria dos percursos pedonais (e. g. construção de passeios ou marcação de passagens pedonais).

Contudo, a adoção destas medidas deve ser racional do ponto de vista de gestão de recursos e eficaz do ponto de vista do cumprimento do seu objetivo: redução da velocidade de circulação entre os ambientes rural e urbano. Assim, as características das zonas de transição devem induzir nos condutores a prática de velocidades adequadas que permitam que o atravessamento destas zonas e das zonas urbanas subsequentes seja seguro e sustentável, quer para os utentes da estrada, quer para as atividades desenvolvidas à sua margem.

Por isso, o objetivo deste trabalho consiste no desenvolvimento de uma metodologia de avaliação dos impactos das características macroscópicas do ambiente periurbano na velocidade praticada pelos condutores. À aplicação da metodologia proposta está subjacente a recolha de variáveis microscópicas, sobre as quais é efetuado um tratamento estatístico que permite a sua agregação em variáveis macroscópicas representativas da geometria da estrada e do ambiente envolvente. Posteriormente, é efetuada uma análise de regressão que permite concluir sobre os efeitos relativos dos diversos fatores que caracterizam a estrada - geometria, ambiente e sinalização de velocidade limite - nas velocidades praticadas em zonas periurbanas.

A metodologia proposta neste artigo é ainda aplicada a um caso de estudo, constituído por um conjunto de 27 locais situados em estradas de duas vias ${ }^{4}$ em Portugal, nos quais foi conduzida uma campanha de recolha de velocidades e de variáveis microscópicas caracterizadoras da geometria e do ambiente das estradas. Observou-se ainda a presença de sinalização de limite legal de velocidade especificamente adotada nos locais.

Pretende-se que as conclusões deste estudo possam auxiliar projetistas e entidades gestoras da rede viária na consideração das medidas mais úteis para a prática de velocidades adequadas em zonas periurbanas. A implementação deste tipo de medidas ocorre, muitas vezes, em fase de exploração da rede viária, sempre que é necessário de intervencionar zonas de transição com elevada sinistralidade ou caso a expansão urbana provoque alterações nos limites das zonas periurbanas. Nesta fase, há que averiguar se as características da estrada e da sua envolvente são, por si só, suficientes para que os condutores reduzam a sua velocidade. E, em caso negativo, é necessário definir o campo de atuação mais eficaz.

Devido à natureza dos locais envolvidos, tem-se dado uma maior ênfase ao tratamento de zonas periurbanas de estradas existentes. No entanto, as conclusões deste estudo podem ser úteis em fase de definição do traçado. Por outras palavras, se um projetista é incumbido de elaborar uma estrada com uma determinada velocidade base, deve ponderar as possíveis soluções geométricas e ambientais (caso haja corredores alternativos que atravessem diferentes ambien-

\footnotetext{
${ }^{2}$ rotatórias (terminologia brasileira).

${ }^{3}$ lombadas (terminologia brasileira).

${ }^{4}$ faixas de tráfego (terminologia brasileira).
}

tes) que resultem num traçado que induza a prática dessa mesma velocidade. Para tal, o conhecimento do impacto dos diversos fatores na velocidade é também importante.

\section{METODOLOGIA PROPOSTA}

\subsection{Seleção de locais e variáveis}

A aplicação da metodologia proposta pressupõe a recolha de informação sobre um conjunto de variáveis microscópicas representativas das características dos locais considerados para avaliação. A amostra de locais em análise deve ser o mais alargada possível, de modo a garantir a fiabilidade dos resultados. Nesses locais, devem ser recolhidas as velocidades dos veículos (em secção) e diversos indicadores relacionados com a geometria das estradas e com o ambiente envolvente às mesmas. Pretende-se que as velocidades obtidas sejam representativas das zonas de transição onde os locais de recolha se inserem, pelo que se deve optar pela escolha de locais situados apenas em retas ${ }^{5}$ independentes e correspondentes ao seu ponto médio. Uma reta é considerada independente caso a sua extensão seja suficiente para que a velocidade desejada por $85 \%$ dos condutores seja atingida. Uma reta é ainda considerada independente caso a sua extensão permita alguma aceleração, mesmo sem nela ser possível atingir a referida velocidade, devido à obrigação de redução de velocidade por motivos de segurança na abordagem à curva seguinte. Os locais em curva são preteridos, de modo a evitar que o valor do raio seja o fator condicionante na escolha de velocidade dos condutores.

Outro contributo para que os indicadores recolhidos sejam representativos das zonas de transição prende-se com a recolha de características geométricas do trecho a montante do elemento alvo de medição de velocidades. Para a delimitação desse trecho, dois critérios podem ser adotados: (i) extensão fixa, definindo, por exemplo, uma extensão de 1 $\mathrm{km}$ contada a partir do local de recolha de velocidades. (ii) extensão variável, considerando a curva e a reta anteriores ao local de levantamento de velocidades, a exemplo do caso de estudo apresentado na secção seguinte do presente artigo. Caso se opte por este último critério, um cuidado extra deve ser atendido na escolha das retas em análise: não devem ser selecionadas retas demasiado extensas que permitam a dissipação do efeito das respetivas curva e reta anteriores nas velocidades praticadas pelos condutores. A consideração do trecho a montante em relação à secção de medição resulta na individualização das observações não só por local, como também por sentido de circulação, o que pode ser benéfico para o aumento da dimensão da amostra.

Desta forma, sugere-se a recolha das seguintes variáveis microscópicas referentes aos locais a estudar:

- Variável recolhida nas retas em análise:

- Velocidade de circulação dos veículos em regime livre $[\mathrm{km} / \mathrm{h}]$.

- Variáveis recolhidas nos trechos a montante:

- Raio da curva anterior [m];

- Extensão da curva anterior [m]; e

- Extensão da reta anterior [m].

- Variáveis recolhidas nas retas em análise e trechos a montante:

- Largura média das vias [m];

${ }^{5}$ tangentes (terminologia brasileira) 
- Largura média das bermas ${ }^{6}[\mathrm{~m}]$;

- Desobstrução lateral média [m];

- Declividade $[\mathrm{m} / \mathrm{km}]$;

- Densidade de construções marginais [n. $\left.{ }^{\circ} / \mathrm{km}\right]$;

- Densidade de pontos de conflito [n. $\left.{ }^{\circ} / \mathrm{km}\right]$;

- Densidade de passeios $[\mathrm{m} / \mathrm{km}]$;

- Variáveis binárias caracterizadoras do tipo de ocupação do solo: residencial, comercial e industrial; e

- Variáveis binárias representativas da existência de sinalização de limite legal de velocidade: 50 $\mathrm{km} / \mathrm{h}, 70 \mathrm{~km} / \mathrm{h}$ ou outro.

A velocidade de circulação em regime livre define-se pela velocidade praticada pelos condutores quando não circulam restringidos pela presença do veículo precedente (AASHTO, 2004). Assim, a velocidade reflete a resposta dos condutores face às características da estrada e do ambiente envolvente. Neste estudo, sugere-se a recomendação de Lobo et al. (2011), segundo a qual um veículo circula em regime livre quando o intervalo temporal para o veículo precedente (gap) é igual ou superior a 6 segundos. A velocidade em regime livre para cada observação é determinada pelo percentil 85 do perfil de velocidades pontuais dos veículos que apresentam um gap mínimo de 6 segundos, observado na secção média das retas em análise.

A largura das bermas e a desobstrução lateral consideradas resultam da média dos valores representativos para o trecho em análise, medidos à esquerda e à direita. A desobstrução lateral representa a distância entre a guia lateral e um objeto fixo existente à margem da estrada.

Os efeitos do perfil longitudinal na velocidade não são tão marcantes quanto os efeitos do perfil transversal e do traçado em planta, revelando-se mais expressivos para a classe minoritária dos veículos pesados. Opta-se então pela sua caraterização apenas através de um indicador agregado, a declividade, correspondente ao somatório das diferenças de cotas entre sucessivos pontos altos e baixos incluídos no trecho, dividido pela extensão do mesmo.

As variáveis expressas em forma de densidade são observadas em ambas as margens da estrada, no conjunto das retas em análise e respetivos trechos a montante. A densidade de construções marginais traduz o número de construções por quilómetro, a densidade de pontos de conflito representa o número de intersecções e passagens pedonais por quilómetro e a densidade de passeios expressa a extensão de passeio (em metros) existente por quilómetro de estrada.

Relativamente à tipologia de uso do solo envolvente à estrada, considera-se como situação de base o uso residencial. Devem, por isso, ser criadas variáveis binárias para representação de outras tipologias (e.g. comercial ou industrial). Estas variáveis adotam o valor 1 sempre que no trecho é dominante a correspondente utilização do solo, assumindo o valor 0 quando tal não se verifica.

Num procedimento análogo, devem ser criadas variáveis binárias para cada limite legal de velocidade imposto pela sinalização vertical, diferente da situação de base de 90 $\mathrm{km} / \mathrm{h}$ que se verifica na ausência de qualquer sinalização. Estas variáveis assumem o valor 1 na presença do limite correspondente, ou o valor 0 no caso contrário.

\footnotetext{
${ }^{6}$ acostamentos (terminologia brasileira).
}

\subsection{Análise multivariada}

A metodologia proposta com vista à avaliação dos impactos da geometria da estrada, do ambiente rodoviário e do limite legal de velocidade na velocidade em regime livre praticada pelos condutores em zonas de transição contempla três etapas: (i) agregação das variáveis originais em variáveis macroscópicas; (ii) formulação numérica das variáveis macroscópicas; (iii) avaliação dos efeitos das diferentes variáveis na velocidade em regime livre. Estas etapas recorrem, respetivamente, a três métodos estatísticos de análise de dados multivariados: classificação hierárquica (análise de clusters), análise em componentes principais e análise de regressão.

A velocidade de circulação dos veículos em regime livre, como variável dependente do estudo, é apenas considerada na análise de regressão. Também as variáveis referentes ao uso do solo e à existência de sinalização de limite legal de velocidade são unicamente utilizadas nessa etapa, uma vez que a sua tipologia binária não permite a inclusão no tratamento estatístico das etapas anteriores.

\subsubsection{Agregação das variáveis originais em variáveis macroscópicas}

Tendo como objetivo encontrar grupos de variáveis semelhantes, recorre-se a métodos de classificação hierárquica (agregação ascendente) sobre o conjunto de variáveis. Para medida de proximidade entre as variáveis, utiliza-se o coeficiente de correlação de Pearson. Para critério de agregação entre classes, usam-se três medidas diferentes (Gordon, 1999):

- Ligação pela menor distância (single linkage): a distância entre duas classes A e B é dada pelo menor valor de distância entre um elemento de A e um elemento de $\mathrm{B}$, tal como se representa na Equação 1:

$$
D(A, B)=\min \{d(a, b), a \in A \wedge b \in B\}
$$

- Ligação pela maior distância (complete linkage): a distância entre duas classes A e B é dada pelo maior valor de distância entre um elemento de A e um elemento de B, conforme se expressa na Equação 2:

$$
D(A, B)=\max \{d(a, b), a \in A \wedge b \in B\}
$$

- Ligação pela maior distância (complete linkage): a distância entre duas classes A e B é dada pelo maior valor de distância entre um elemento de A e um elemento de B, conforme se expressa na Equação 2:

$$
D(A, B)=\frac{1}{n_{A} n_{B}} \sum_{\substack{a \in A \\ b \in B}} d(a, b)
$$

em que,

$n_{A}$ : cardinal da classe A; e

$n_{B}$ : cardinal da classe B.

Os dendrogramas resultantes da aplicação destes métodos permitem verificar a agregação das variáveis microscópicas em diferentes classes, que podem ser interpretadas como características macroscópicas das zonas periurbanas das estradas sob avaliação. Então, o número de classes a reter nesta fase não deverá ser demasiado elevado - tipicamente duas a três classes - procurando-se realçar a dicotomia entre características geométricas e ambientais, sem prejuízo da 
perspetiva macroscópica do estudo.

Os dendrogramas obtidos para os três critérios de agregação poderão refletir algumas diferenças na classificação das variáveis microscópicas. A escolha de um destes dendrogramas, necessária para a implementação das etapas seguintes, pode ter em conta procedimentos mais teóricos ou apenas empíricos.

\subsubsection{Formulação numérica das variáveis macroscópicas}

Na segunda etapa, utiliza-se o método de análise em componentes principais (Saporta, 1990) com o objetivo da formulação numérica das variáveis macroscópicas provenientes da etapa anterior. Para cada uma destas variáveis, é extraída a primeira componente principal a partir das variáveis microscópicas que formam o respetivo grupo. Assim, cada característica macroscópica passa a ser representada por uma combinação linear de variáveis originais, conforme se traduz na Equação 4.

$$
M_{j}=\sum_{i} w_{i j} m_{i j}
$$

em que,

$M_{j}$ : variável macroscópica $j$;

$m_{i j}$ : variável microscópica $i$ agregada na variável macroscópica $j$; e

$w_{i j}$ : peso da variável microscópica $i$ na variável macroscópica $j$.

Como os quadros de dados a tratar por esta metodologia são tendencialmente heterogéneos, deve-se proceder à normalização das variáveis originais previamente à aplicação da análise em componentes principais.

\subsubsection{Avaliação dos efeitos das diferentes variáveis na velocidade em regime livre}

Na terceira e última etapa, com vista à avaliação dos efeitos das variáveis macroscópicas na velocidade em regime livre, recorre-se a uma análise de regressão, traduzida pela Equação 5.

$$
v 85=f(M)+\varepsilon
$$

em que,

v85: velocidade de circulação dos veículos em regime livre;

$f(M)$ : função do conjunto das variáveis macroscópicas; e $\varepsilon$ : erro com distribuição normal.

Para $f(M)$ sugerem-se os modelos linear, exponencial e potencial. Uma análise dos valores de significância das variáveis macroscópicas e dos coeficientes de correlação ajuda a escolha do modelo que melhor se ajusta ao conjunto de dados.

\section{CASO DE ESTUDO}

\subsection{Descrição dos dados}

Os locais e variáveis utilizados no presente caso de estudo provêm da base de dados construída pelos autores para a modelação da velocidade em regime livre em estradas de duas vias e dois sentidos. Esta base de dados é fruto de uma vasta campanha de recolha, levada a cabo em estradas situadas no norte de Portugal, no âmbito do projeto
SAFESPEED, financiado pela Fundação para a Ciência e a Tecnologia.

Nesta campanha, a velocidade dos veículos em secção foi aferida com recurso a contadores de tráfego, contendo um sensor de radar Doppler, uma memória de dados Flash RAM e um relógio em tempo real, que registam o momento de passagem de cada veículo (hh:mm:ss), a respetiva velocidade e o gap em relação ao veículo precedente. Os contadores devem ser colocados na secção média dos elementos alvo de levantamento e a sua presença deve ser o mais dissimulada possível, pois os condutores tendem a abrandar sempre que avistam objetos desconhecidos instalados à margem da estrada. As recolhas de velocidades foram realizadas em condições diurnas, de pavimento seco e de não congestionamento. Os períodos de recolha tiveram uma duração variável, sendo que, para cada observação, foi garantida a obtenção de velocidades de um mínimo de 100 veículos livres, seguindo a recomendação do Highway Capacity Manual 2000 (TRB, 2000) para efeitos de estimativa de velocidade operacional.

As características do traçado em planta foram obtidas a partir da recolha de pontos $(x, y)$ efetuada com recurso ao veículo instrumentado do Laboratório de Análise de Tráfego da Faculdade de Engenharia da Universidade do Porto. Neste veículo, foi instalado um sistema de GPS que, para maior precisão na tomada de dados, recebe correções diferenciais enviadas por estações fixas. O ajuste dos elementos do traçado (retas e curvas) aos pontos recolhidos foi posteriormente realizado com recurso ao software Autodesk Civil 3D 2010. As características geométricas referentes ao perfil transversal, bem como a inclinação longitudinal, foram medidas in situ com recurso a métodos topográficos de levantamento expedito.

Para aplicação da metodologia proposta, foi então selecionado um conjunto de 27 locais em reta (total de 54 observações, considerando os dois sentidos), pertencentes às estradas estudadas no âmbito do SAFESPEED. Estas estradas são pautadas pelo elevado número de zonas periurbanas, com presença de construção marginal dispersa. A irregularidade do terreno manifesta-se através da elevada sinuosidade do traçado, com muitas curvas de raio inferior a 100 $\mathrm{m}$, mas nem por isso sujeito a inclinações longitudinais significativas, que se mantêm geralmente abaixo dos $6 \%$.

Tendo-se optado pela utilização de dados pré-existentes em detrimento de um levantamento específico, esta escolha obriga a uma adaptação das variáveis microscópicas face à lista proposta em 2.1, o que constitui uma limitação deste caso de estudo. Assim, consideraram-se as seguintes variáveis:

- Variáveis recolhidas nas retas em análise:

- v85: velocidade de circulação dos veículos em regime livre $(\mathrm{km} / \mathrm{h})$;

- $\quad l v$ : largura das vias [m];

- $\quad l b$ : largura das bermas [m];

- dl: desobstrução lateral [m]; e

- $\quad$ i: inclinação longitudinal [\%].

- Variáveis recolhidas nos trechos a montante:

- rca: raio da curva anterior [m];

- eca: extensão da curva anterior [m]; e

- era: extensão da reta anterior [m].

- Variáveis recolhidas nas retas em análise e trechos a montante:

- dcm: densidade de construções marginais 
$\left[\mathrm{n} .{ }^{\circ} / \mathrm{km}\right] ;$

- $\quad d p c$ : densidade de pontos de conflito [n. $\% / \mathrm{km}]$;

- $\quad d p$ : densidade de passeios $[\mathrm{m} / \mathrm{km}] ; \mathrm{e}$

- slv: existência de sinalização de limite legal de velocidade (uma variável binária).

A primeira adaptação consiste nas larguras de vias e bermas e na desobstrução lateral, aqui consideradas como os valores representativos das retas em análise, em vez da totalidade dos trechos. Também a declividade dos trechos foi substituída pela inclinação longitudinal nas retas alvo de levantamento de velocidades, facto que não introduz grandes perturbações na aplicação da metodologia, dadas as reduzidas inclinações observadas nas estradas do caso de estudo.

Relativamente ao uso marginal do solo, sendo predominantemente residencial em todos os locais aferidos (situação de base), não implicou a criação de qualquer variável binária.

Quanto à existência de sinalização de limite legal de velocidade, verifica-se que, das 46 observações com limite legal de velocidade restringido pela sinalização, 42 estão sujeitas a um limite de $50 \mathrm{~km} / \mathrm{h}$ e apenas quatro apresentam um limite de $70 \mathrm{~km} / \mathrm{h}$. Face a esta disparidade no número de observações, optou-se pela utilização de uma única variável binária que assume o valor 1 quando um desses limites de velocidade é estabelecido pela sinalização vertical para o trecho em análise. Caso contrário, a variável assume o valor 0 .

Por último, refira-se aquela que talvez seja a maior limitação deste caso de estudo: não é possível garantir que a recolha de velocidades tenha sido efetuada exclusivamente em retas independentes. Embora a extensão média se cifre nos 345 m, o desvio padrão atinge os 200 m, o que resulta num coeficiente de variação de $58 \%$.

Na Tabela 1, são apresentadas algumas medidas descritivas das variáveis microscópicas consideradas na aplicação da metodologia ao caso de estudo.

Também da observação da Tabela 1 ressalta a elevada dispersão de algumas variáveis, o que confirma a reduzida homogeneidade das características dos locais em análise.

\subsection{Efeitos das características macroscópicas na velocidade em regime livre}

Os métodos de tratamento estatístico utilizados na aplicação das três etapas da metodologia ao caso de estudo foram e- xecutados com o auxílio do software SPSS, versão 17.0 (Maroco, 2003).

Na primeira etapa, a utilização dos três métodos de classificação hierárquica sobre o conjunto de variáveis microscópicas ( $l v, l b, d l, i, r c a$, era, eca, $d c m, d p c$ e $d p)$ produziu classes semelhantes para os mesmos níveis de desagregação. Este facto contribui para validar a estrutura em classes apresentada e verificar a dicotomia entre dois grupos principais de características macroscópicas das zonas periurbanas: geometria e ambiente. Na Figura 1, apresenta-se apenas o dendrograma resultante do critério de agregação pela ligação média entre classes.

Da análise da Figura 1, verifica-se que, com exceção da variável $i$, todas as restantes se agrupam em classes representativas das características macroscópicas da estrada. Se forem consideradas duas variáveis macroscópicas, estas apresentam a seguinte constituição:

- Geometria da estrada (geo): agrupa $l b, d l, l v$, eca, era e rca; e

- Ambiente $(a m b)$ : agrupa $d c m, d p c$ e $d p$.

Sem prejuízo de uma perspetiva macroscópica, o dendrograma mostra que, à custa da divisão da classe caracterizadora da geometria da estrada em dois subgrupos, é possível uma agregação das variáveis originais em três variáveis macroscópicas:

- Perfil transversal na reta (ptr): agrupa $l b, d l$ e $l v$;

- Traçado a montante (tm): agrupa eca, era e rca; e

- Ambiente ( $a m b)$ : agrupa $d c m, d p c$ e $d p$.

Quer a retenção de duas, quer de três variáveis macroscópicas foram estudadas na segunda etapa da metodologia. Uma superior desagregação das variáveis seria nefasta à perspetiva macroscópica que se pretende para este estudo, uma vez que daí resultariam mais classes formadas apenas por uma variável original. De facto, conforme foi anteriormente referido, esta situação já se verifica em relação à variável $i$ logo a partir do segundo nível de desagregação. No entanto, por ser a única variável que representa uma outra característica distinta da geometria da estrada - o perfil longitudinal - é a que, globalmente, menos se encontra correlacionada com as restantes variáveis. Devido a estas considerações, a variável $i$ não foi incluída na segunda etapa da metodologia, entrando diretamente na análise de regressão.

Tabela 1. Média, desvio padrão e coeficiente de variação das variáveis selecionadas

\begin{tabular}{|c|c|c|c|}
\hline Variáveis & Média & Desvio padrão & Coeficiente de variação \\
\hline \multicolumn{4}{|l|}{ Recolhidas na reta } \\
\hline$v 85[\mathrm{~km} / \mathrm{h}]$ & 74 & 9 & $13 \%$ \\
\hline $\operatorname{lv}[\mathrm{m}]$ & 3,48 & 0,17 & $5 \%$ \\
\hline $\mathrm{lb}[\mathrm{m}]$ & 1,47 & 1,11 & $75 \%$ \\
\hline$d l[\mathrm{~m}]$ & 1,72 & 1,08 & $63 \%$ \\
\hline$i[\%]$ & 0,0 & 3,2 & \\
\hline \multicolumn{4}{|c|}{ Recolhidas no trecho a montante } \\
\hline $\operatorname{rca}[\mathrm{m}]$ & 199 & 163 & $82 \%$ \\
\hline eca $[\mathrm{m}]$ & 105 & 81 & $78 \%$ \\
\hline $\operatorname{era}[\mathrm{m}]$ & 232 & 372 & $160 \%$ \\
\hline \multicolumn{4}{|c|}{ Recolhidas na reta e trecho a montante } \\
\hline$d c m\left[\mathrm{n} .{ }^{\circ} / \mathrm{km}\right]$ & 23 & 17 & $71 \%$ \\
\hline$d p c\left[\mathrm{n} .^{\circ} / \mathrm{km}\right]$ & 5 & 3 & $70 \%$ \\
\hline$d p[\mathrm{~m} / \mathrm{km}]$ & 276 & 299 & $108 \%$ \\
\hline
\end{tabular}




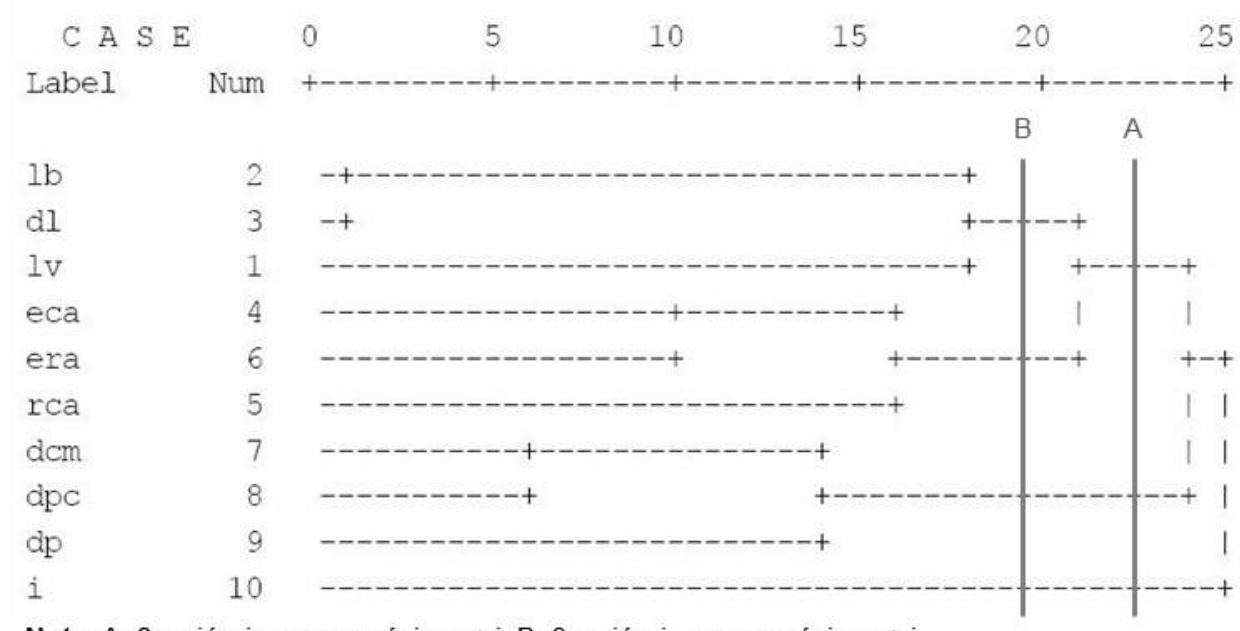

Nota. A: 2 variáveis macroscópicas + i; B: 3 variáveis macroscópicas + $\mathrm{i}$

Figura 1. Dendrograma com utilização da ligação média entre classes (SPSS)

Desta forma, procedeu-se na segunda etapa à formulação numérica das variáveis macroscópicas originadas na primeira etapa com recurso ao método de análise em componentes principais. Executou-se a prévia normalização das variáveis microscópicas (opção incluída no software SPSS).

$\mathrm{Na}$ Tabela 2, apresenta-se os pesos e a percentagem da variância total explicada relativa à primeira componente principal obtida para as variáveis geo e $a m b$.

Na Tabela 3, estão representados os pesos e a percentagem da variância total explicada da primeira componente principal estimada para as variáveis $p t r, t m$ e $a m b$.

Constata-se que a divisão das características geométricas em duas outras variáveis macroscópicas (ptr e tm) acarreta vantagens ao nível da percentagem da variância total explicada, uma vez que o seu valor mais baixo sobe de $42 \%$ para $63 \%$. Por isso, apenas esta solução agregativa de variáveis é considerada na análise de regressão da terceira etapa.

Da análise da Tabela 3, verifica-se ainda que a primeira componente principal associada à variável macroscópica ptr é fortemente influenciada pelos pesos das variáveis $l b$ e $d l$. $\mathrm{O}$ menor peso de $l v$ pode ser explicado pela menor variabilidade da largura das vias face à largura das bermas e à desobstrução lateral nas estradas portuguesas de duas vias, facto que pode ser observado na Tabela 1 .
As restantes variáveis microscópicas presentes na Tabela 3 contribuem de forma significativa e com pesos semelhantes para as primeiras componentes principais dos respetivos grupos.

Na terceira e última etapa, avaliaram-se os efeitos das variáveis $p t r, t m, a m b, i$ e $s v l$ na velocidade em regime livre através de uma análise de regressão. A forma linear revelou um bom ajuste às variáveis envolvidas, encontrando-se os respetivos resultados na Tabela 4 .

O coeficiente de correlação linear de 0,657 expressa uma correlação considerável entre as variáveis (Cohen, 1988). Consideraram-se as variáveis independentes normalizadas (com exceção da variável binária svl), de modo a permitir a comparação entre si dos coeficientes de regressão e a consequente influência relativa na variável dependente (v85).

Na análise dos pressupostos do modelo de regressão linear, considera-se os aspetos associados à multicolinearidade das variáveis independentes e à análise dos resíduos: homocedasticidade, normalidade e independência. Na análise da multicolinearidade, determina-se os valores das estatísticas VIF e de tolerância, sendo que o valor mais baixo da estatística que avalia o limite de tolerância é de 0,853 e o maior valor de VIF é de 1,193.

Tabela 2. Primeira componente principal das variáveis geo e amb

\begin{tabular}{ll}
\hline Variáveis & Primeira componente principal \\
\hline Variável macroscópica: geo & \\
Variáveis microscópicas: & \\
lv & 0,536 \\
Ib & 0,796 \\
dl & 0,767 \\
rca & 0,528 \\
eca & 0,662 \\
era & 0,557 \\
\% variância explicada & $42 \%$ \\
\hline Variável macroscópica: $a m b$ & \\
Variáveis microscópicas: & \\
$d c m$ & 0,877 \\
$d p c$ & 0,895 \\
$d p$ & 0,726 \\
\% variância explicada & $70 \%$
\end{tabular}


Tabela 3. Primeira componente principal das variáveis ptr, tm e amb

\begin{tabular}{cc}
\hline Variáveis & Primeira componente principal \\
\hline Variável macroscópica: $p t r$ & \\
Variáveis microscópicas: & \\
$l v$ & 0,512 \\
$l b$ & 0,973 \\
$d l$ & 0,949 \\
\% variância explicada & $70 \%$ \\
\hline Variável macroscópica: $t m$ & \\
Variáveis microscópicas: & \\
rca & 0,700 \\
eca & 0,887 \\
era & 0,788 \\
\% variância explicada & $63 \%$ \\
\hline Variável macroscópica: $a m b$ & \\
Variáveis microscópicas: & \\
$d c m$ & 0,877 \\
$d p c$ & 0,895 \\
$d p$ & 0,726 \\
\% variância explicada & $70 \%$ \\
\hline
\end{tabular}

Tabela 4. Resultados da regressão linear

\begin{tabular}{lcl}
\hline Variável & Coeficiente de regressão & Valor $\boldsymbol{p}$ \\
\hline Constante & 78,347 & 0,000 \\
$p t r$ & 1,402 & 0,214 \\
$t m$ & 3,764 & 0,001 \\
$a m b$ & $-2,850$ & 0,014 \\
$i$ & $-1,504$ & 0,150 \\
svl & $-5,494$ & 0,089 \\
\hline Coeficiente de correlação $(R)$ & 0,657 & \\
Coeficiente de determinação $\left(R^{2}\right)$ & 0,431 & \\
\hline
\end{tabular}

No que respeita à análise dos resíduos, calcula-se o valor da estatística de Durbin-Watson, realiza-se vários gráficos de resíduos (Figura 2) e efetua-se o teste de KolmogorovSmirnov para avaliar a adequabilidade da distribuição normal aos resíduos. Este teste conduziu a um valor p de 0,634. O valor encontrado para a estatística de Durbin-Watson foi $d=1,235$. Já os valores de $d_{L}\left(d_{\text {inferior }}\right)$ e $d_{U}\left(d_{\text {superior }}\right)$ são, respetivamente, 1,37 e 1,77 . O valor obtido de $d$ sugere uma ligeira autocorrelação positiva entre os resíduos. Com exceção do teste de Durbin-Watson, todos os procedimentos indicam que o modelo de regressão é adequado.

Da análise da Tabela 4, constata-se, em primeiro lugar, que a natureza dos efeitos (positiva ou negativa) que as diferentes variáveis exercem na velocidade livre de circulação está de acordo com a que seria intuitivamente expectável para cada uma das características da estrada analisadas e com o referido por SWOV (2008). Deste modo, têm impactos positivos na velocidade em regime livre o incremento da envergadura do perfil transversal e a melhoria da qualidade geométrica do traçado a montante. Em contraponto, o agravamento da inclinação longitudinal reduz as velocidades praticadas, assim como o agravamento do ambiente rodoviário, à medida que o atrito lateral se vai aproximando das características de ambiente urbano.

É também possível verificar que as características do traçado a montante tendem a ser significativamente mais preponderantes para a variação da velocidade em regime livre do que o perfil transversal e a inclinação longitudinal no elemento de medição. Isto indicia que a experiência recente sobre o traçado (referente ao trecho a montante) seja mais
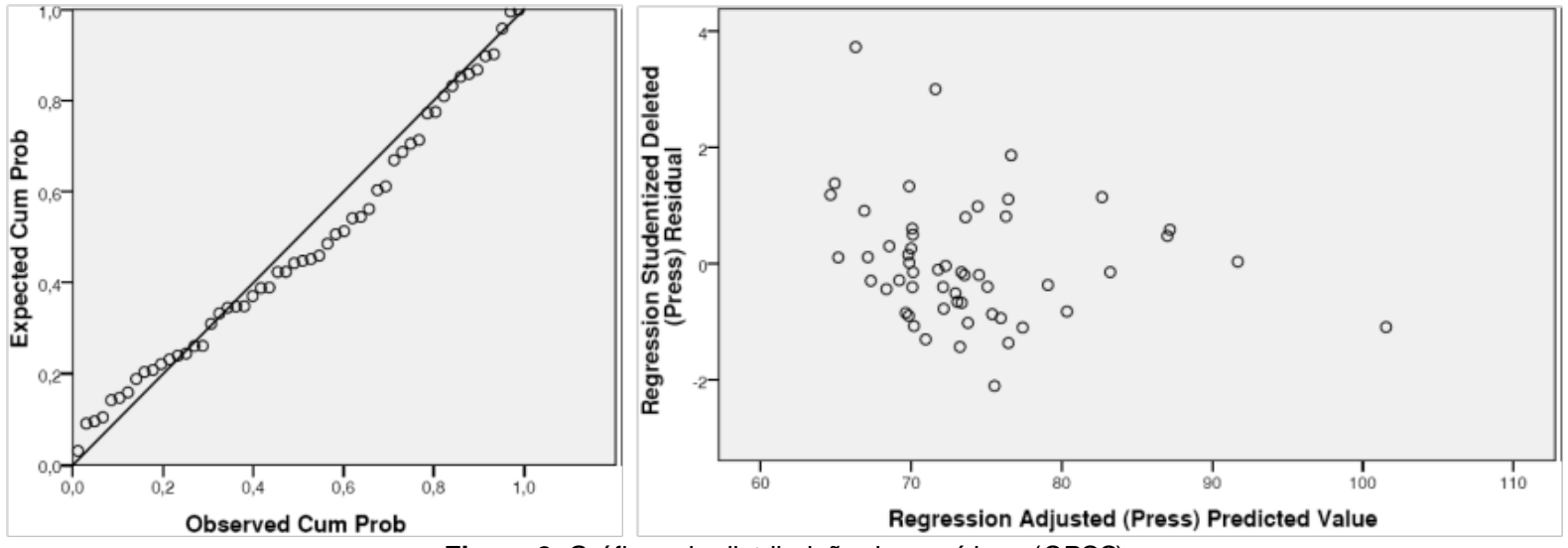

Figura 2. Gráficos de distribuição dos resíduos (SPSS) 
relevante para a escolha de velocidade face à experiência imediata de condução. Ressalve-se, no entanto, que os valores $\mathrm{p}$ associados às variáveis ptr e $i$ se situam além do nível de significância de $10 \%$ normalmente associado a estudos do género, facto a que poderá não ser alheia a relativamente reduzida dimensão da amostra.

A magnitude dos efeitos induzidos pelo ambiente onde a estrada se insere situa-se num patamar intermédio entre os efeitos causados pelas características da reta em análise e pelas características do traçado a montante.

Finalmente, a interpretação do coeficiente da variável não normalizada ( $s v l)$ é a mais direta e intuitiva. No conjunto dos locais analisados, sempre que se observa a presença de um sinal vertical que limita a velocidade a um valor inferior aos $90 \mathrm{~km} / \mathrm{h}$ estabelecidos pelo Código de Estrada, a velocidade em regime livre é reduzida em cerca de $5 \mathrm{~km} / \mathrm{h}$, valor que está de acordo com o referido por County Surveyors’ Society (1994) e SWOV (2008).

\section{CONCLUSÕES}

A velocidade de circulação em regime livre adotada por um condutor, não dependendo da influência do restante tráfego, sofre impactos significativos causados por dois grandes grupos de características inerentes à estrada: geometria e ambiente envolvente. Fazendo uso da metodologia proposta neste artigo, é possível estudar a influência destas características macroscópicas nas velocidades praticadas em ambiente periurbano. A aplicação da metodologia ao caso de estudo confirma a sua adequabilidade para a avaliação dos referidos efeitos, mesmo em presença de algumas limitações impostas pela natureza dos dados utilizados.

Deste modo, esta aplicação resulta na agregação das variáveis microscópicas recolhidas em variáveis macroscópicas que realçam a dicotomia entre geometria e ambiente. A caracterização geométrica, quando comparada com a ambiental, envolve um superior número de variáveis recolhidas que apresentam níveis de correlação mais baixos entre si. Assim, veio-se a constatar que, para a avaliação dos impactos da geometria na velocidade livre, seria profícua a agregação das variáveis originais em duas variáveis macroscópicas: uma caracterizadora do perfil transversal e outra caracterizadora do traçado em planta a montante. Como vantagens desta agregação, refere-se:

- A maior percentagem de variância explicada pela primeira componente principal extraída de cada grupo de variáveis e representativa da característica macroscópica associada ao grupo; e

- A mais fácil comparação entre a eficácia de adoção de medidas ao nível do perfil transversal e do traçado em planta, por via à obtenção da velocidade de circulação pretendida.

No caso de estudo, verifica-se então que a qualidade geométrica do traçado em planta a montante produz um impacto na velocidade bastante superior aos impactos produzidos pela envergadura do perfil transversal e pela inclinação longitudinal do elemento de medição. Isto denota que a velocidade é mais influenciada pela experiência recente de condução, que gera no condutor expectativas relativamente ao traçado, do que pelas condições do local onde é aferida. Então, de modo a que a velocidade praticada pelos condutores nas zonas periurbanas estudadas seja inferior à veloci- dade adotada em ambiente rural e seja adequada à aproximação do ambiente urbano, deve ser equacionada, em primeiro lugar, a hipótese de aumento da sinuosidade do traçado à entrada e ao longo das zonas de transição. Em estradas existentes, esta medida é normalmente preconizada pela construção de rotundas, devido aos impactes reduzidos que produzem sobre terrenos e construções marginais. Menos eficazes que as alterações ao traçado em planta, a redução do perfil transversal e o agravamento da inclinação longitudinal dos trainéis têm efeitos na velocidade similares entre si, embora esta última medida seja de difícil execução prática em estradas já construídas. Outras alterações ao perfil longitudinal, enquadradas nas medidas de acalmia de tráfego, tais como a construção de lombas ou de passagens pedonais elevadas, não são analisadas por não constituírem objetivo específico deste estudo.

Em relação ao ambiente envolvente à estrada, verifica-se que o seu agravamento causa na velocidade uma redução de magnitude intermédia, comparativamente com os efeitos produzidos pela redução das características geométricas do traçado em planta do trecho a montante e do perfil transversal. No entanto, em estradas já construídas, a atuação a este nível está praticamente limitada à construção de passeios e à implementação de passagens pedonais, dado que o incremento da densidade de construção depende das políticas de expansão urbanística e o aumento do número de interseções está subjacente à construção de novas estradas.

Quanto à imposição de um limite legal de velocidade inferior a $90 \mathrm{~km} / \mathrm{h}$ por via da instalação de sinalização vertical, esta medida produz uma redução de velocidade de 5 $\mathrm{km} / \mathrm{h}$ no conjunto das velocidades praticadas pelos condutores. Não sendo possível a comparação direta com os efeitos provocados pelas restantes variáveis recolhidas, refira-se que esta redução corresponde a cerca de $7 \%$ da velocidade livre média observada para o conjunto das observações e a apenas $12,5 \%$ a $25 \%$ da redução de velocidade imposta pela adoção de limites de $50 \mathrm{~km} / \mathrm{h}$ e $70 \mathrm{~km} / \mathrm{h}$, respetivamente. Deste modo, pode-se constatar a reduzida relevância deste tipo de sinalização na velocidade praticada pelos condutores nas zonas periurbanas estudadas. Importa ainda salientar que nas estradas estudadas não se verificou uma significativa incidência de fiscalização e controlo de velocidade.

Os autores consideram que a base de dados que suporta o caso de estudo do presente artigo não é suficientemente ampla para que as respetivas conclusões possam ser extrapoladas para o âmbito nacional ou internacional. Por isso, a aplicação futura da metodologia proposta a um conjunto mais alargado de locais, incorporando características de estradas de outros países, seria útil para a avaliação, numa perspetiva mais abrangente, da influência das características macroscópicas das zonas de transição nas velocidades praticadas pelos condutores.

\section{AGRADECIMENTOS}

Os autores agradecem à Fundação para a Ciência e a Tecnologia, pelo financiamento providenciado no âmbito do projecto SAFESPEED PTDC/TRA/72998/2006.

\section{REFERÊNCIAS BIBLIOGRÁFICAS}

AASHTO (2004) A Policy on Geometric Design of Highways and Streets ( $5^{\mathrm{a}}$ ed.). American Association of State Highway and Transportation Officials, Washington, D.C., USA. 
Cohen, J. (1998) Statistical Power Analysis for the Behavioral Sciences ( $2^{\mathrm{a}}$ ed.). Lawrence Erlbaum Associates Inc., Hillsdale, NJ, USA.

County Surveyors' Society (1994) Village Speed Control Working Group - Final Report. Department for Transport Scottish and Welsh Office and Transport Research Laboratory, UK.

Denton, G. G. (1976) The Influence of Adaptation on Subjective Velocity for an Observer in Simulated Rectilinear Motion. Ergonomics, v. 19, p. 409-430.

Gordon, A. D. (1999) Classification (2 $2^{\mathrm{a}}$ ed.). Chapman \& Hall/CRC, Boca Raton, FL, USA.

Lobo, A.; M. A. P. Jacques; C. M. Rodrigues e A. Couto (2011) Free-Gap Evaluation for Two-Lane Rural Highways. Transportation Research Record, v. 2223, p. 9-17.

Maroco, J. (2003) Análise Estatística com utilização do SPSS (2ª ed.). Edições Sílabo, Lisboa, Portugal.

Matthews, M. L. (1978) A Field Study of the Effects of Drivers' Adaptation to Automobile Velocity. Human Factors, v. 20, p. 709-716.

NCHRP (2011) Speed Reduction Techniques for Rural High-to-Low Speed Transitions. NCHPR Synthesis 412, National Cooperative Highway Research Program, Transportation Research Board of the National Academies, Washington, D.C., USA.

Saporta, G. (1990) Probabilités analyse des données et statistique. Editions TECHNIP, Paris, France.

Schmidt, F. e J. Tiffin (1969) Distortion of Drivers' Estimates of Automobile Speed as a Function of Speed Adaptation. Journal of Applied Psychology, v. 53, p. 536-539.

SWOV (2008) Speed Choice: the influence of man, vehicle, and road. Institute for Road Safety Research, Leidschendam, the Netherlands.

TRB (2000) Highway Capacity Manual 2000. Transportation Research Board of the National Academies, Washington, D.C., USA.

Tziotis, M. (1992) Crashes on the Approaches to Provincial Cities. Report No. 31, Monash University Accident Research Centre, Victoria, Australia. 